\title{
A NEW PROOF OF THE EQUIVALENCE OF E. H. MOORE*
}

BY J. P. BALLANTINE

The theorem which is here called the equivalence of $\mathrm{E}$. $\mathrm{H}$. Moore is a statement of a necessary and sufficient condition for the existence of a solution of a system of linear equations.

In Moore's own proof of this equivalence, there is a hypothesis to the effect that if the Hermitian square of a vector $\alpha^{\prime}$ is zero, all the elements are zero. That is,

$$
\alpha^{\prime} \bar{\alpha}_{1}=0 \cdot \supset \cdot \alpha^{\prime}=0^{\prime} .
$$

In plain language, take a set of complex numbers, or numbers in any number system, multiply each by its conjugate, and add; then if the sum is zero, all the original numbers are zero.

This hypothesis is satisfied in many number systems, and holds in every case in which Moore used his equivalence. Unfortunately, the writer $\dagger$ used the equivalence in the number system of residues modulo 2 . For such a number system the hypothesis does not hold, as for example in the case of the vector $\alpha^{\prime}=(1,1)$, where $\alpha^{\prime} \bar{\alpha},=1+1 \equiv 0$ (modulo 2 ). To form a logical basis for this work, it was necessary to have a proof, such as the following, of the equivalence which would not use the limiting hypothesis.

A general system of linear equations is written

$$
\alpha_{\prime}^{\prime \prime} \xi_{\prime \prime}=\eta,
$$

in matrix form. The prime range $\left({ }^{\prime}\right)$ and the second range $\left({ }^{\prime}\right)$ are conceptually distinct, so that the matrix $\alpha_{,}^{\prime \prime}$ may be square or not. Let $n$ be the number of rows in the matrix, or the number of equations in the system, and $m$ the number of columns, or unknowns.

The symbol $\exists$ is read "there exists," and $\ni$ is read "such that." The statement that a solution of the system of equations exists is written

* Presented to the Society, August 30, 1932.

$\dagger A$ postulational introduction to the four color problem, Six Studies in Mathematics, University of Washington Publications, 1930, vol. 2, No. 1, p. 9. 


$$
\exists \xi_{\prime \prime} \ni \alpha_{\prime \prime}^{\prime \prime} \xi_{\prime \prime}=\eta_{\prime} .
$$

Two vectors $\alpha^{\prime}$ and $\beta$, are said to be orthogonal if

$$
\alpha^{\prime} \beta,=0 \text {. }
$$

A vector $\alpha^{\prime}$ is orthogonal to all the columns of a matrix $\beta_{, \prime \prime}$ if

$$
\alpha^{\prime} \beta_{,}^{\prime \prime}=0^{\prime \prime} \text {. }
$$

It may be shown by straightforward substitution that

$$
X \cdot \supset \cdot Y,
$$

where $Y$ is the proposition

$$
\gamma^{\prime} \alpha_{,}^{\prime \prime}=0^{\prime \prime} \cdot \supset \cdot \gamma^{\prime} \eta_{,}=0 .
$$

That $X$ implies $Y$ and, conversely, $Y$ implies $X$, constitutes the equivalence of $\mathrm{E}$. H. Moore.* In Moore's proof of the converse, he normalizes the columns of the matrix, and for this step he needs the hypothesis referred to earlier. The following proof does not require this hypothesis.

To prove that $Y$ implies $X$, assume $Y$ true and $X$ false. Then the proof is complete if we are led to a contradiction.

Commutative multiplication is assumed here, but the proof can easily be extended to a noncommutative number system. Unique division, except by zero, is also assumed.

A set, $\Gamma_{m}$, is built up of vectors $\gamma_{1, m}, \gamma_{2, m}, \cdots, \gamma_{N, m}$ which, together with all their linear combinations, will include all possible vectors orthogonal to all the columns of $\alpha_{\prime}^{\prime \prime}$. There appears to be no obvious way to write down the set $\Gamma_{m}$ at one step, but it can be done by mathematical induction. Two sequences of sets of vectors are formed

$$
\begin{aligned}
& A_{0}, A_{1}, \cdots, A_{m}, \\
& \Gamma_{0}, \Gamma_{1}, \cdots, \Gamma_{m} .
\end{aligned}
$$

$A_{k}$ is the set of vectors consisting of the first $k$ columns of $\alpha_{1}^{\prime \prime}$, and $\Gamma_{k}$ is a set of vectors which, together with all their linear combinations, includes all the vectors orthogonal to all the vectors of $A_{k}$.

* E. H. Moore, Lectures on General Analysis. Memoirs of the American Philosophical Society, vol. 1, 1935. 
Since $A_{0}$ is a null set, the vectors of $\Gamma_{0}$ may be written down, by inspection, as the rows of Hilbert's unit matrix $\delta^{\prime}$, namely,

$$
\Gamma_{0}=\left(\gamma_{1,0}^{\prime}, \gamma_{2,0}^{\prime}, \cdots, \gamma_{n, 0}^{\prime}\right)
$$

where

$$
\gamma_{1,0}^{\prime}=1,0,0, \cdots, 0, \quad \gamma_{2,0}^{\prime}=0,1,0, \cdots, 0, \quad \cdots .
$$

The formation of $\Gamma_{(k+1)}$ from $\Gamma_{k}$ is as follows. All the vectors of $\Gamma_{k}$ are assumed orthogonal to all those of $A_{k}$. Now $A_{(k+1)}$ involves one new vector $\nu$, not in $A_{k}$. To see if all the vectors of $\Gamma_{k}$ are accidentally orthogonal also to $\nu_{1}$, we consider the following $N_{k}$ numbers:

$$
Q_{j}=\gamma_{j, k}^{\prime} \nu^{\prime}, \quad\left(j=1,2, \cdots, N_{k}\right),
$$

where $N_{k}$ is the number of vectors $\gamma_{1, k}, \cdots, \gamma_{N, k}$ in $\Gamma_{k}$.

CASE 1. Now if all the numbers $Q_{j}$ are zero, then all the vectors of $\Gamma_{k}$ are orthogonal to all the vectors of $A_{(k+1)}$. This will happen, of course, if $\nu$, the $(k+1)$ st column of $\alpha_{\prime}^{\prime \prime}$ is linearly dependent on the preceding columns. It may, as far as we have yet shown, happen in other cases.

CASE 2. All the numbers $Q_{j}$ are not zero, so that $Q_{J} \neq 0$, where $J$ is some particular value of $j$. Let $P$ denote the reciprocal of $Q_{J}$. In our number system such reciprocals are supposed to exist uniquely. As a tentative definition of $\Gamma_{(k+1)}$, consider

$$
\left[\gamma_{j, k+1}^{\prime}\right] \equiv\left[\gamma_{j, k}^{\prime}-Q_{j} P \gamma_{J, k}^{\prime}\right]
$$

To test that all the vectors $\gamma_{i,(k+1)}^{\prime}$ are orthogonal to all the vectors of $A_{(k+1)}$, note that it is obvious for the first $k$ of the latter. In the case of $\nu_{1}$,

$$
\begin{aligned}
\gamma_{j,(b+1)}^{\prime} \nu_{1} & =\gamma_{j, b}^{\prime} \nu_{,}-Q_{j} P \gamma_{J, b}^{\prime} \nu_{1} \\
& =Q_{j}-Q_{j} P Q_{J}=0,
\end{aligned}
$$

by the definition of $P$. In testing the linear independence of the vectors of $\Gamma_{(k+1)}$ as defined above, we find that one of them, $\gamma_{J,(k+1)}^{\prime}$, is identically zero. That one must be deleted, and the others constitute the set $\Gamma_{(k+1)}$ as finally defined, so that the number of vectors in $\Gamma_{(k+1)}$ is one less than in $\Gamma_{k}$, or 


$$
N_{(k+1)}=N_{k}-1 \text {. }
$$

The linear independence of the $N_{k}-1$ vectors of $\Gamma_{(k+1)}$ follows directly from the linear independence of the vectors of $\Gamma_{k}$. Thus the rank of $\Gamma_{k}$ is $N_{k}$, the same as the number of vectors in it. Let the rank of $A_{k}$ be $R_{k}$. Then in Case 1,

$$
N_{(k+1)}=N_{k}, \quad R_{(k+1)}=R_{k}, \quad \text { or } \quad R_{k}+1,
$$

according as $\nu$, is or is not linearly dependent on the vectors of $A_{k}$. In Case 2,

$$
N_{(k+1)}=N_{k}-1, \quad R_{(k+1)}=R_{k}+1 .
$$

Therefore, in both cases,

$$
N_{(k+1)}+R_{(k+1)} \geqq N_{k}+R_{k} .
$$

When $k=0, N_{0}=n=$ the number of rows in $\alpha_{\prime}^{\prime \prime}$ and $R_{0}=0$; so that $N_{0}+R_{0}=n$, and therefore $N_{k}+R_{k} \geqq n$.

Now prolong the sequence $A_{0}, \cdots, A_{m}$, by defining $A_{(m+1)}$ as the set of all columns of $\alpha_{1}^{\prime \prime}$ and the vector $\eta$, Then $\Gamma_{(m+1)}$ is formed from $\Gamma_{m}$ in the manner described for forming $\Gamma_{(k+1)}$ from $\Gamma_{k}$.

To obtain the contradiction, we are assuming $Y$ true and $X$ false. Since $Y$ is true, all the vectors of $\Gamma_{m}$ are orthogonal to all the vectors of $A_{(m+1)}$, so we have Case 1 , where $\Gamma_{(m+1)}=\Gamma_{m}$. Therefore $N_{(m+1)}=N_{m}$.

Since $X$ is false, the last vector of $A_{(m+1)}$ is not expressible linearly in terms of the vectors of $A_{m}$. Therefore $R_{(m+1)}=R_{m}+1$, so that

$$
N_{(m+1)}+R_{(m+1)}>N_{m}+R_{m} \geqq n .
$$

Now the sets $A_{(m+1)}$ and $\Gamma_{(m+1)}$ are mutually orthogonal, and the vectors of those sets have only $n$ elements. It is, therefore, impossible for the sum of their ranks to exceed $n$, and we have the desired contradiction.

Or, by extending the sequence of $A$ 's further, one could obtain as a contradiction a single set of vectors in $n$-space with rank $n+1$. This, of course, could be reduced step by step to a set of vectors in one dimension of rank two.

The University of Washington 